\title{
Energy budget analysis of aeroelastic limit-cycle oscillations
}

\author{
A.C.L.M. van Rooij ${ }^{\mathrm{a}, *}$, J. Nitzsche ${ }^{\mathrm{a}}$, R.P. Dwight ${ }^{\mathrm{b}}$ \\ ${ }^{a}$ Institute of Aeroelasticity, German Aerospace Center (DLR), Bunsenstrasse 10, 37073 Göttingen, Germany \\ ${ }^{b}$ Faculty of Aerospace Engineering, Delft University of Technology, Kluyverweg 1, 2629 HS Delft, The Netherlands
}

\begin{abstract}
In this work limit-cycle oscillations (LCOs) caused by aerodynamic non-linearities were analysed using fluid-structure interaction (FSI) simulations of a two-degree-of-freedom airfoil system. A comparison with sinusoidal forced motion oscillation simulations at the fundamental frequency was performed. The results show that using the first harmonic component for the forced motion oscillations is a good approach to describe the limit-cycle oscillations considered in this work. Following the work of G. Dietz, G. Schewe and H. Mai (2004), Journal of Fluids and Structures, Vol. 19, an energy budget analysis of the limit-cycle oscillations was performed. Power analysis of the LCO reveals that the power of the lift and moment show non-linear behaviour with increasing amplitude. The linearised equivalent power components that would occur in case of flutter were computed. These show that the defect in the power of the lift in the non-linear case is caused by the increase of the phase of the lift with oscillation amplitude, which is the result of the unsteady shock wave motions on both upper and lower surface of the airfoil. The power of the moment also shows a defect, which is much smaller than in case of the lift. This defect is caused by variations in both the magnitude and the phase of the moment with oscillation amplitude.
\end{abstract}

Keywords:

Limit-cycle oscillations, Aerodynamic non-linearities, Fluid-structure interaction, Complex mode shape, Power analysis

\section{Introduction}

The onset of flutter can be predicted by linearised stability analysis. In contrast, to predict limit-cycle oscillations, non-linearities in the aeroelastic system need to be taken into account. Hence, the equations of motion can no longer be linearised. The non-linearities that cause the amplitude limitation of aeroelastic oscillations can be either structural or aerodynamic in nature. At the German Aerospace Center (DLR) in Göttingen several measurement campaigns have been conducted with a two-degree-of-freedom (DOF) system with the supercritical NLR7301 airfoil (e.g. [1, $2,3,4,5]$ ) in which limit-cycle oscillations were observed. Shock-induced separation and trailing-edge separation were considered to be the amplitude limiters. Euler simulations with this same supercritical airfoil have shown that the changing type of shock motion (from Tijdeman type A to type B) can also be a cause for (nested) LCOs [6, 7]. Numerous other numerical investigations have also studied LCOs of airfoils with aerodynamic non-linearities (e.g. $[8,9,10,11])$. However, the global mechanism responsible for the amplitude limitation of these two-degree-offreedom LCOs has not been identified yet.

To study the causes of amplitude limitation, Dietz et al. [4] conducted wind tunnel experiments in which an unstable aeroelastic 2-DOF system was forced to oscillate at several constant amplitudes by the use of a flutter controlsystem. The oscillations were prevented from growing or decaying in amplitude by applying an additional excitation

(c) 2016. This manuscript version is made available under the CC-BY-NC-ND 4.0 license https://creativecommons.org/licenses/ by-nc-nd/4.0/

Publisher's version can be found at: http://dx.doi.org/10.1016/j.jfluidstructs.2016.11.016

*Corresponding author

Email addresses: Anouk.vanRooij@dlr.de (A.C.L.M. van Rooij), Jens.Nitzsche@dlr.de (J. Nitzsche), R.P.Dwight@tudelft.nl (R.P. Dwight)

Postprint accepted by Journal of Fluids and Structures 
force on the heave DOF. This variable-gain additional force was proportional to the heave velocity and effectively acted as a viscous damping element. At the LCO amplitude the exciter force did not do any work to the system. At constant-amplitude oscillations smaller than the LCO amplitude, energy was extracted from the system, whereas at oscillation amplitudes larger than the LCO amplitude, energy had to be fed into the system. It is important to note, that with this experimental set-up the viscous damping was modified slightly, whereas the eigenfrequencies and complex-valued mode shapes of the coupled aeroelastic system remained (largely) intact. In a post-processing step the contributions of several components (damping, aerodynamic lift, aerodynamic moment) to the total power fed into or extracted from the system were analysed for (forced) constant-amplitude oscillations at several amplitudes. At the (natural) LCO amplitude the total power, i.e. the sum of aerodynamic power and structural damping power averaged over one oscillation cycle, was zero, as expected. Both the power of the aerodynamic lift and moment were found to be weakly non-linear with amplitude, leading to a nearly glancing intersection of the total power curve with the zero axis [4]. Furthermore, Dietz et al. [4, 5] observed that the phase difference between heave and pitch that they found during the (forced) LCO experiments was small enough (in the order of $5-10^{\circ}$ ) such that the LCOs they observed can be seen as a single degree-of-freedom motion with its rotation point upstream of the airfoil. Bendiksen [12] also observed that for LCOs close to the linear flutter boundary, the mode shape resembles a single-DOF pitching motion.

Although Dietz et al. [4] performed a good account of work on analysing the energy budget of limit-cycle oscillations of a 2-DOF system, several questions remain unanswered. E.g. why does the total power behave in the way it does, i.e. why do we have a nearly glancing intersection of the total power with the zero axis? And what are the causes of this, i.e. what global and local features are responsible for this? In order to answer these questions, an energy budget analysis is applied in this work using numerical simulations. Following standard-procedure, fluid-structure-coupled simulations of a 2-DOF airfoil system above the flutter boundary were performed. Furthermore, forced motion oscillation simulations were performed at several constant oscillation amplitudes. In both cases the averaged aerodynamic power components were calculated on the basis of the aerodynamic forces and the motion data. To find the causes for the amplitude limitation, the deviations of these power components with respect to the linearised (flutter) case are analysed and the sources of aerodynamic non-linearity are traced back. Although several investigations have used local power or work distributions to study limit-cycle oscillations e.g. $[6,5,7,13]$, little work has been performed on the global energy budget of limit-cycle oscillations. This work bridges between the initial work done on the global energy budget of LCOs by Dietz et al. [4] and the local approaches of [6, 5, 7, 13]. However, the goal of this work is not to validate the numerical results against the wind tunnel experiments performed at German Aerospace Center $[1,2,3,4,5]$. Therefore, no attempt is made to directly compare the results obtained here with those of Dietz et al. $[4,5]$. Only indirect comparisons of the energy budget will be made.

Section 2 of this paper describes the computational methods used in this work. In Section 3 the results of the forced motion oscillation simulations and the FSI simulations are presented and analysed. Finally, conclusions are drawn.

\section{Computational methods}

This section describes the computational methods and set-up used in the current investigation. First, the CFD code will be briefly discussed. Second, the structural model is addressed. Then, the fluid-structure coupling and the LCO modelling using forced motion oscillations are presented. Finally, the results of a mesh- and timestep convergence study are presented.

\subsection{CFD code and set-up}

The Computational Fluid Dynamics (CFD) code used is the DLR-TAU code developed by the German Aerospace Center [14]. This code is a finite-volume, cell-vertex-based, unstructured compressible RANS solver. For spatial discretisation a 2nd order central scheme [15] is used. Temporal discretisation is realised by dual time stepping [16] with the 2nd order accurate Backward Differencing Formula (BDF2) integration scheme for the physical time. The turbulence model used for closure is the Menter SST model.

The airfoil used in this study is the supercritical NLR7301 airfoil with a blunt trailing edge (coordinates were taken from [17]). Its design Mach number is 0.721 and its design lift coefficient is 0.595 . The Reynolds number with respect to the chord length was set to $2 \cdot 10^{6}$ for all CFD simulations. The chord length of the airfoil is $0.3 \mathrm{~m}$. In order 


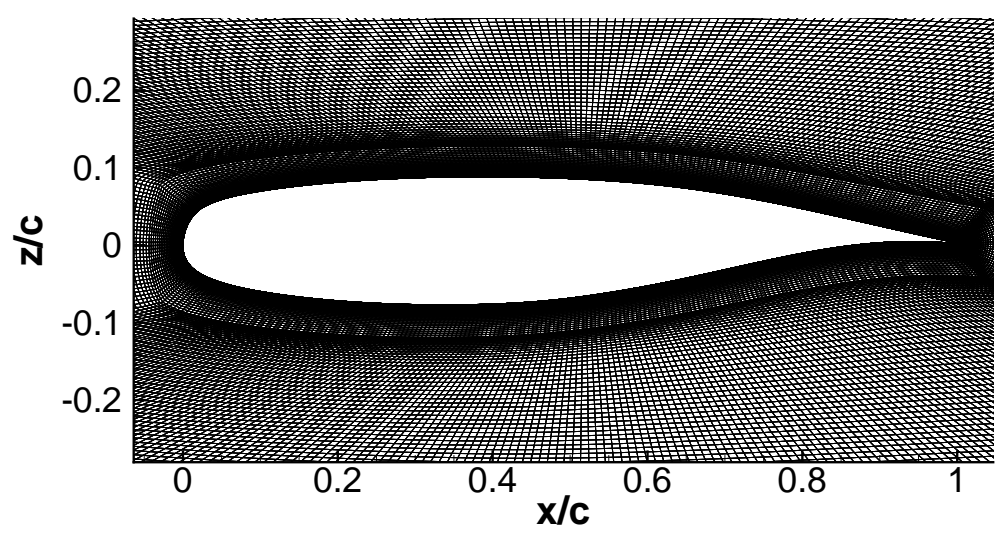

Figure 1: Mesh used for the CFD computations (medium)

\begin{tabular}{lc} 
Structural parameter & Value \\
\hline \hline Wing span $b$ & $1.0 \mathrm{~m}$ \\
Chord length $c$ & $0.3 \mathrm{~m}$ \\
Mass $m$ & $26.268 \mathrm{~kg}$ \\
Mass moment of inertia (about the elastic axis) $I_{\alpha}$ & $0.079 \mathrm{kgm}$ \\
Torsional spring constant $K_{\alpha}$ & $6.646 \cdot 10^{3} \mathrm{Nm} / \mathrm{rad}$ \\
Bending spring constant $K_{h}$ & $1.078 \cdot 10^{6} \mathrm{~N} / \mathrm{m}$ \\
Static moment (related to EA) $S_{\alpha}$ & $0.331 \mathrm{kgm}$ \\
Pitch damping $D_{\alpha}$ & $0.0687 \mathrm{kgm}{ }^{2} / \mathrm{s} / \mathrm{rad}$ \\
Plunge damping $D_{h}$ & $45.764 \mathrm{~kg} / \mathrm{s}$ \\
\hline
\end{tabular}

Table 1: Structural parameters

to simulate a two-dimensional airfoil a straight three dimensional wing is used, with symmetry planes at both sides. In this work a wing with a span of $1.0 \mathrm{~m}$ is used. The unstructured O-type mesh consisting of hexahedrons that has used for all CFD simulations, has 65888 points. The non-dimensional first cell height $y^{+}$is smaller than 1 . The mesh has one cell in span-wise direction. Figure 1 shows a zoom of the mesh near the airfoil.

The motion of the airfoil is achieved by a rigid-body displacement of the complete grid. In this way the grid cells keep their form and no grid deformation in the classical sense is necessary. The additional fluxes that are introduced by displacing the complete grid are taken into account such that the geometric conservation law is satisfied.

The airfoil was tested at various angles of attack and at $M=0.74$. The static temperature has been set to $273 \mathrm{~K}$. The flow is fully turbulent in all simulations.

\subsection{Structural model}

In this work the airfoil is modelled using a linear structural model with two degrees of freedom (pitch and plunge). The elastic axis is located at the quarter-chord point. A schematic representation of the aeroelastic system system is shown in Figure 2. The structural properties of the two-degree-of-freedom system have been taken from the windtunnel model of the NLR7301 airfoil used by Dietz et al. [4]. They are depicted in Table 1.

\subsection{Fluid-structure interaction simulations}

The equations of motion of the system depicted in Figure 2 are:

$$
\mathbf{M} \vec{q}+\mathbf{D} \vec{q}+\mathbf{K} \vec{q}=\vec{f},
$$




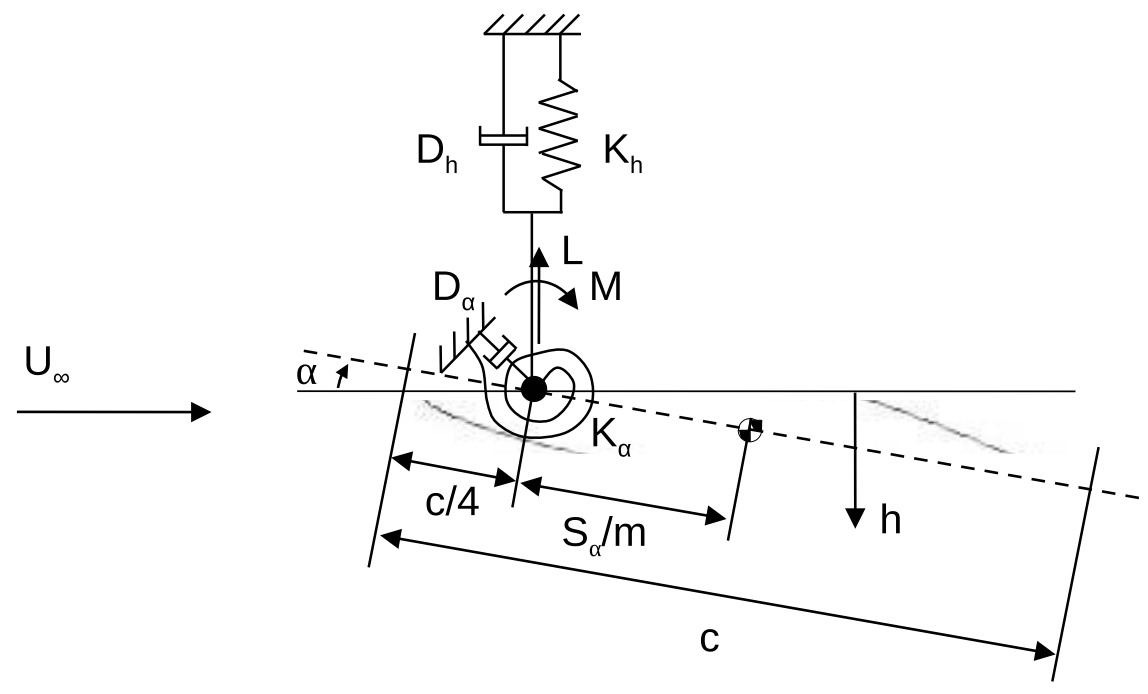

Figure 2: Sketch of the model with two degrees of freedom

where the mass matrix $\mathbf{M}$, damping matrix $\mathbf{D}$, stiffness matrix $\mathbf{K}$, force vector $\vec{f}$ the displacement vector $\vec{q}$ are defined as:

$$
\begin{gathered}
\mathbf{M}=\left[\begin{array}{cc}
m & S_{\alpha} \\
S_{\alpha} & I_{\alpha}
\end{array}\right], \mathbf{D}=\left[\begin{array}{cc}
D_{h} & 0 \\
0 & D_{\alpha}
\end{array}\right], \\
\mathbf{K}=\left[\begin{array}{cc}
K_{h} & 0 \\
0 & K_{\alpha}
\end{array}\right], \vec{f}=\left[\begin{array}{c}
-L \\
M
\end{array}\right]=\left[\begin{array}{c}
-\left(\tilde{L}-L_{0}\right) \\
\tilde{M}-M_{0}
\end{array}\right] \text { and } \vec{q}=\left[\begin{array}{l}
h \\
\alpha
\end{array}\right]=\left[\begin{array}{c}
\tilde{h}-h_{0} \\
\tilde{\alpha}-\alpha_{0}
\end{array}\right] .
\end{gathered}
$$

Here $L$ is the lift, $M$ the aerodynamic moment and $h$ and $\alpha$ are the plunge displacement and pitch angle, respectively. In order to trim the airfoil at a desired mean angle of attack $\alpha_{0}$ (and possibly at a desired plunge position $h_{0}$ ), the steady loads at this desired mean angle of attack $\left(L_{0}, M_{0}\right)$ are subtracted from the actual loads $(\tilde{L}, \tilde{M})$.

The system (1) is solved using fluid-structure coupling, where the pitch angle $\alpha$, plunge displacement $h$, angular velocity $\dot{\alpha}$ and the plunge velocity $\dot{h}$ as well as the resulting forces and moments are exchanged between the CFD code and the structural solver. This is done multiple times during each time step, such that at the end of each time step an equilibrium is achieved. Hence, the FSI coupling as applied here is commonly referred to as "strong". This is in contrast to a so-called weak coupling, where both the fluid and the structure are converged separately and forces and displacements are exchanged after each time step. Strong coupling therefore avoids additional errors due to partitioning. The second order backward differencing formula (BDF2) has been used for the numerical integration of (1).

In order to verify the correct implementation of the FSI coupling algorithm, the energy conservation of the algorithm has to be checked. The mean of the initial energy $E_{0}$ of the two-degree-of-freedom system should remain constant in time according to the energy conservation law. The initial energy is given by:

$$
E_{0}=E_{\mathrm{kin}}+E_{\mathrm{pot}}-W_{\mathrm{nc}} .
$$

Hence, the mean of the total of the kinetic energy $E_{\text {kin }}=1 / 2 \cdot \vec{q}^{T} \mathbf{M} \vec{q}$, the potential energy $E_{\mathrm{pot}}=1 / 2 \cdot \vec{q}^{T} \mathbf{K} \vec{q}$ and the negative of the non-conservative work $W_{\text {nc }}$ should remain constant. The non-conservative work consists of the work done by the structural damping forces and by the aerodynamic loads acting on the airfoil, i.e.:

$$
W_{\mathrm{nc}}=\int_{0}^{t}\left(\vec{q}^{T} \mathbf{D} \vec{q}-\vec{q}^{T} \vec{f}\right) d t .
$$




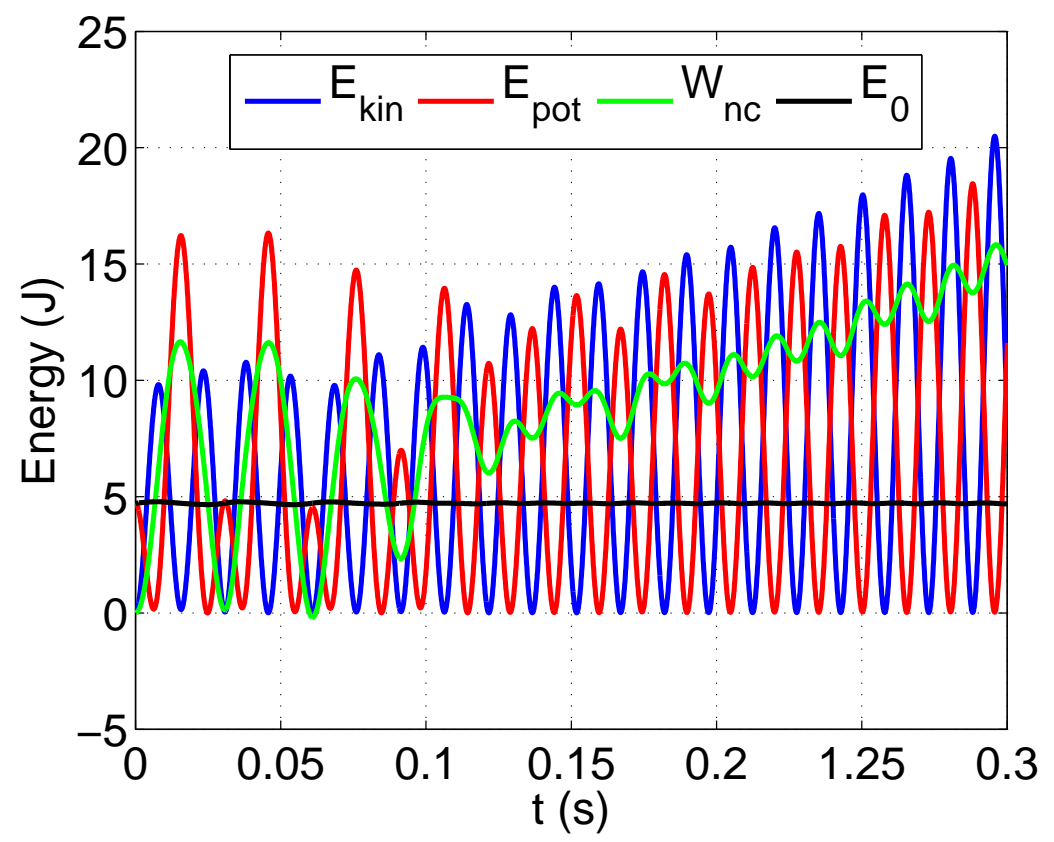

Figure 3: Time evolution of the energy for an FSI simulation

Figure 3 demonstrates that the mean of the initial energy $E_{0}$, as given by (3), is constant during the initial phase of an FSI simulation (see Figure 5 for the complete time signal).

\subsection{Forced motion oscillation simulations}

In the experiments of Dietz et al. [4] the oscillation amplitude is kept constant by applying a flutter-control system, which constrains the oscillations from growing or decaying. This would be similar to constraining the oscillations to grow or decay in a coupled FSI simulation. Here, single-harmonic forced motion oscillations of constant amplitude are used to model such a forced LCO (i.e. an oscillation of constant amplitude restrained by the flutter-control system). In order to do so, the mode shape during the LCO needs to be known, i.e. the frequency, the phase difference between pitch and plunge and the ratio between the pitch and plunge amplitudes are required. This is in contrast to coupled FSI simulations and the wind tunnel experiments of Dietz et al. [4], where the mode shape is implicitly known. Hence, an FSI simulation needs to be performed to find the mode shape of the system. The mode shape during the growth (or decay) of the oscillation amplitude can then be extracted (neglecting the higher harmonics). This information can be used as an input to the forced motion oscillation simulations.

The motion of the airfoil during a forced motion oscillation is prescribed using:

$$
\begin{aligned}
& h(t)=\Delta h \sin \left(\omega t+\phi_{h \alpha}\right), \\
& \alpha(t)=\Delta \alpha \sin \omega t,
\end{aligned}
$$

where $\phi_{h \alpha}$ is the phase difference between pitch and plunge, that is, $\phi_{h \alpha}=\phi_{h}-\phi_{\alpha}$, i.e. $\phi_{h \alpha}$ is the phase of the plunging motion with respect to the pitching motion. Hence, when the phase difference is positive, plunge leads pitch and when $\phi_{h \alpha}$ is negative plunge lags pitch. $\omega$ is the angular frequency.

Note that in (5) and (6) only the first harmonic component is taken into account. It was observed from numerically computed LCOs as well from experimentally obtained LCOs $[4,5]$ that the oscillations are approximately first harmonic. Hence, it is hypothesised that the LCOs observed for the NLR7301 airfoil are purely sinusoidal in the first fundamental frequency. This hypothesis will be tested in Section 3.1. 


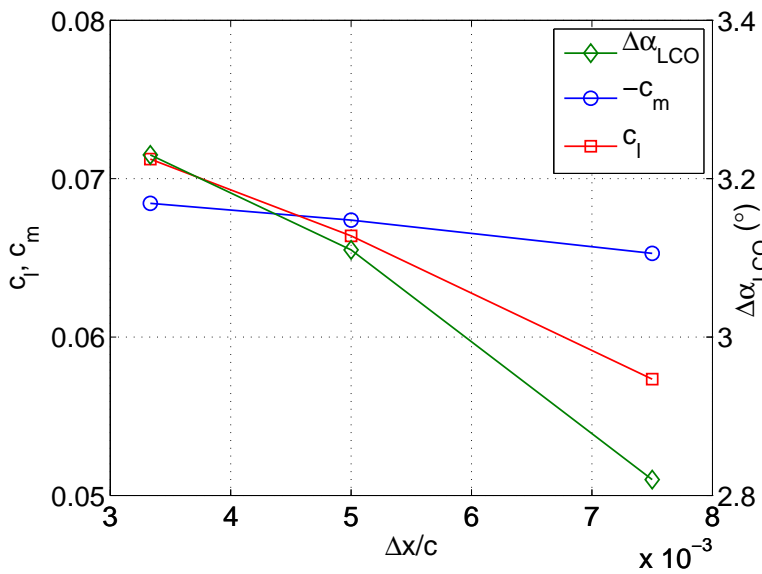

(a) Mesh convergence

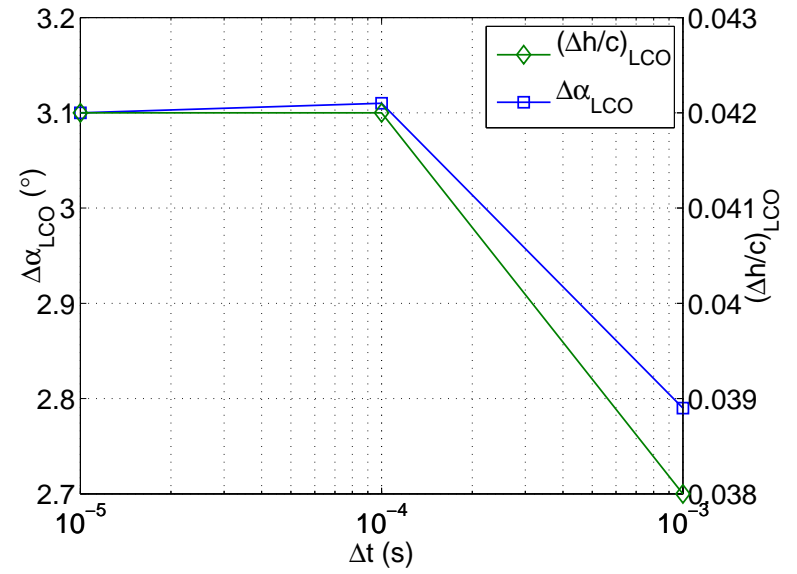

(b) Time step convergence

Figure 4: Mesh and time step convergence for steady $c_{l}$ and $c_{m_{y}}$ and LCO amplitudes

\subsection{Mesh- and time step convergence study}

A mesh- and time step convergence study has been performed for the steady CFD simulations and the FSI simulations. The results are given in Figure 4. Figure 4a shows the results in terms of the steady lift and moment coefficient, $c_{l}$ and $c_{m_{y}}$, respectively and LCO amplitude for three different meshes at $M=0.74, \bar{\alpha}=-0.8^{\circ}$. Note that the moment coefficient has been multiplied by -1 for plotting purposes. The number of points on the airfoil has been increased by a factor of 1.5 between each of the meshes. In addition, the $y^{+}$-value of the first cell is kept at 0.75 .

From Figure $4 \mathrm{a}$ it is observed that all global values are within $15 \%$ of the medium mesh. Furthermore, the deviations of the medium mesh $(\Delta x / c=0.0050)$ with respect to the fine mesh $(\Delta x / c=0.0033)$ are smaller than those to the coarse mesh $(\Delta x / c=0.0075)$, i.e. $7.3 \%$ versus $13.6 \%$ for the lift coefficient, $1.6 \%$ versus $3.1 \%$ for the moment coefficient and $3.9 \%$ versus $9.3 \%$ for the LCO amplitude. Therefore, the medium mesh (with 65888 points) has been used for all simulations (both FSI and forced motion) performed in this work. It was selected as a compromise between accuracy and computational work. Furthermore, the results obtained in this work are only compared to each other and hence a very high resolution of the mesh is not required, since no (direct) validation with experimental results is intended.

The time step $\Delta t$ used for the forced motion simulations as well as for the FSI simulations is $1 \cdot 10^{-4} \mathrm{~s}$. This is equivalent to a non-dimenstional time step size of 0.082 (based on the freestream velocity) or a number of time steps per oscillation periods of 303. For $\Delta t=1 \cdot 10^{-3} \mathrm{~s}$ and $\Delta t=1 \cdot 10^{-5} \mathrm{~s}$, the number of time steps per oscillation period are 29 and 3029, respectively. The results of a time step convergence study are shown in Figure 4b, which compares the LCO amplitude obtained with different time steps at $M=0.74, \bar{\alpha}=-0.8^{\circ}$ (on the medium mesh). As can be seen from this figure the LCO amplitude has a deviation of $10 \%$ or less between the chosen time step sizes, with decreasing difference as the time step is refined further. Therefore, the error made when using a time step of $1 \cdot 10^{-4} \mathrm{~s}$, or 303 timesteps per oscillation period, is acceptable for the investigations in this work.

\section{Results and discussion}

This section shows the results of FSI simulations and the corresponding forced motion oscillation simulations. The results of a power analysis will be shown and discussed for both simulation methods. Furthermore, the first harmonic assumption will be addressed. Then, the effect of oscillation amplitude is studied by comparing the nonlinear aerodynamic forces and power components with their equivalent linearised counterparts. Finally, the global source of the amplitude limitation will be identified. 

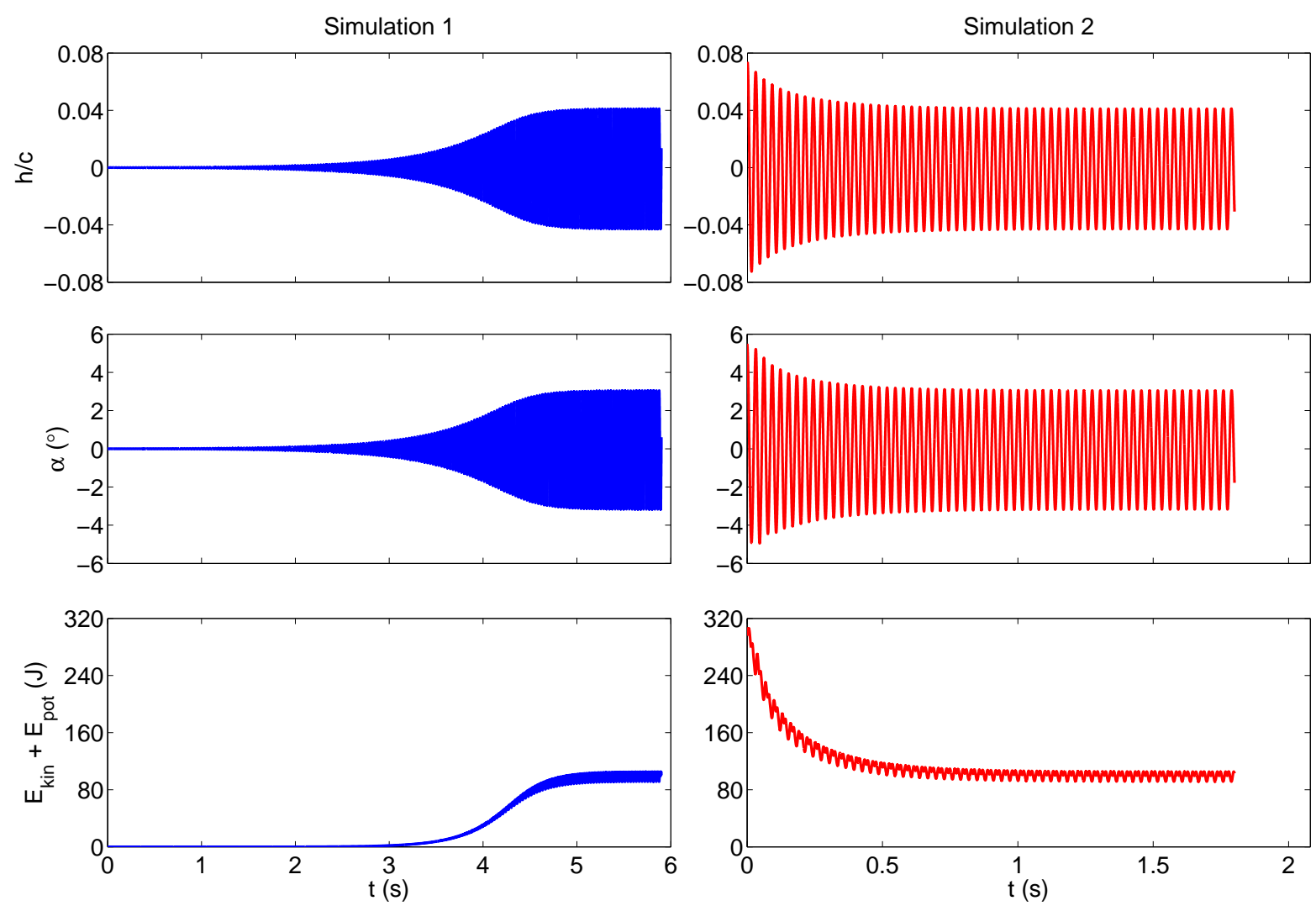

Figure 5: Time evolution of LCO at $M=0.74, \bar{\alpha}=-0.8^{\circ}$ 


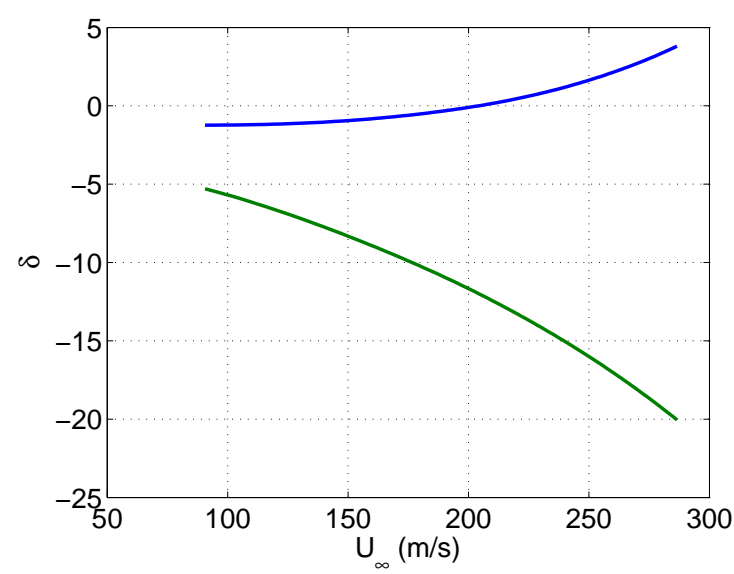

(a) Amplification rate vs. freestream velocity

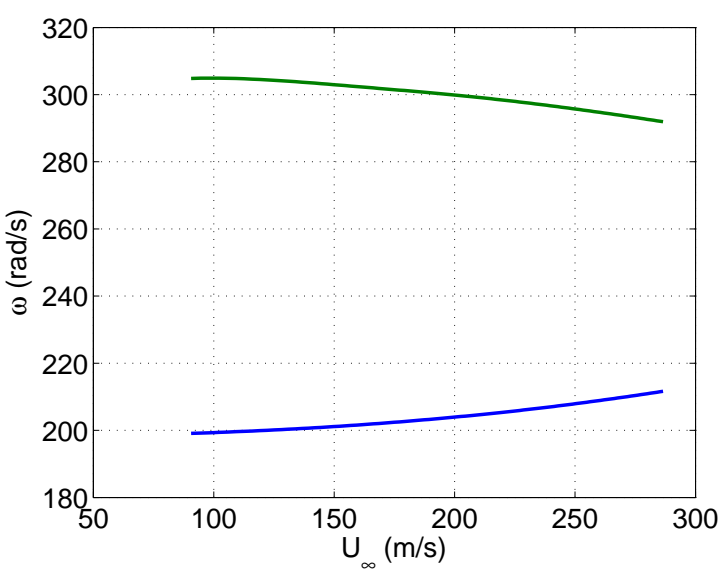

(b) Angular frequency vs. freestream velocity

Figure 6: Amplification rate and angular frequency versus the freestream velocity at $M=0.74, \bar{\alpha}=-0.8^{\circ}$

\subsection{Fluid-structure interaction simulations}

The time evolutions of the pitch angle, plunge displacement and the sum of the kinetic and the potential energy at $M=0.74, \bar{\alpha}=-0.8^{\circ}$ are depicted in Figure 5. The results of two simulations are shown in this figure; on the left-hand side the signals obtained when simulation is started at an amplitude below the LCO amplitude (simulation 1), on the right-hand side the time history obtained when the simulation is started at an amplitude above the LCO amplitude (simulation 2). An LCO amplitude of approximately $3.11^{\circ}$ is observed. The non-dimensional plunge amplitude $\Delta h / c$ is 0.042 . The sum of the kinetic plus the potential energy averaged over one period is observed to be constant.

In order to put the obtained LCO amplitudes in perspective, a linearised flutter analysis using the p-k method $[18]$ in the frequency domain has been performed. Figure 6 shows the amplification rate $\delta$ and the angular frequency $\omega$ versus the freestream speed. A positive amplification rate indicates a motion growing in amplitude, whereas a negative amplification rate indicates a damped motion. The blue line represents the plunging mode and the green line the pitching mode. The plunging mode is seen to become unstable at a velocity of $204.16 \mathrm{~m} / \mathrm{s}$, i.e. this is the flutter velocity at $M=0.74, \bar{\alpha}=-0.8^{\circ}$. Hence, the LCO found here occurs far above the flutter boundary, since the freestream velocity is $245.15 \mathrm{~m} / \mathrm{s}$ for the LCO test case. The amplitude ratio $\left|\theta_{h \alpha}\right|$ at flutter is 0.82 and the phase difference $\phi_{h \alpha}$ at flutter is $4.79^{\circ}$. The reduced frequency $k\left(=\omega c / U_{\infty}\right)$ at flutter is 0.30 .

Figure 7 displays the amplitude ratio $\left|\theta_{h \alpha}\right|$ and the phase difference between pitch and plunge $\phi_{h \alpha}$ versus the oscillation amplitude during simulations 1 and 2. These mode shape parameters were obtained by applying a Fourier transform to the FSI time signals. In order to do so, four oscillation periods were simultaneously considered in one sliding post-processing window. For each post-processing window, a Fourier transform of the time signals was applied to obtain the magnitude and phase of the amplitude ratio. The post-processing windows were overlapping by $95 \%$. The scatter in Figure 7 is caused by this overlap of the post-processing windows. Increasing the number of periods per window or decreasing the overlapping reduces the scatter, but also reduces the number of points at which the amplitude ratio and phase difference are obtained.

The amplitude ratio is seen to remain almost constant over the growth (or decay) in oscillation amplitude. However, the phase difference is observed to change slightly over the oscillation amplitude growth, from about $7.29^{\circ}$ to about $9.46^{\circ}$ at the LCO amplitude. The reduced frequency of the LCO is 0.254 , it does not change during the development towards the LCO. Upon comparing the mode shape at the LCO with the flutter mode shape, it is observed that both $\left|\theta_{h \alpha}\right|$ and $k$ are slightly lower at the LCO. However, $\phi_{h \alpha}$ is larger at the LCO.

The ratio of the higher order harmonic components to the first harmonic component has been computed, see Figure 8. From this figure it is observed that for plunge the ratio is an order of magnitude smaller than for pitch. As the second order harmonic is more than one order of magnitude, or in case of plunge two orders of magnitude, smaller than the 

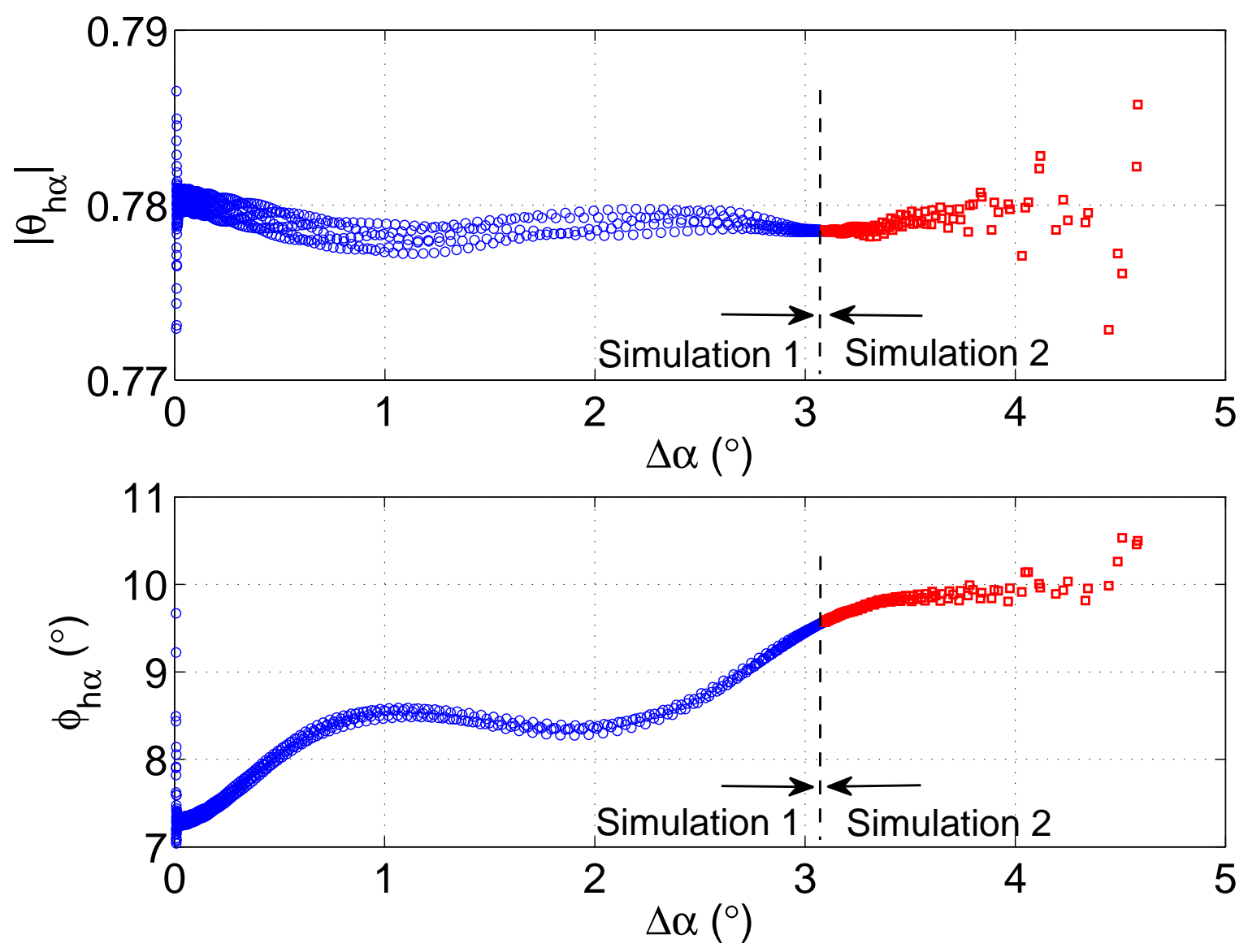

Figure 7: Evolution of the amplitude ratio and phase difference versus the LCO amplitude at $M=0.74, \bar{\alpha}=-0.8^{\circ}$ 


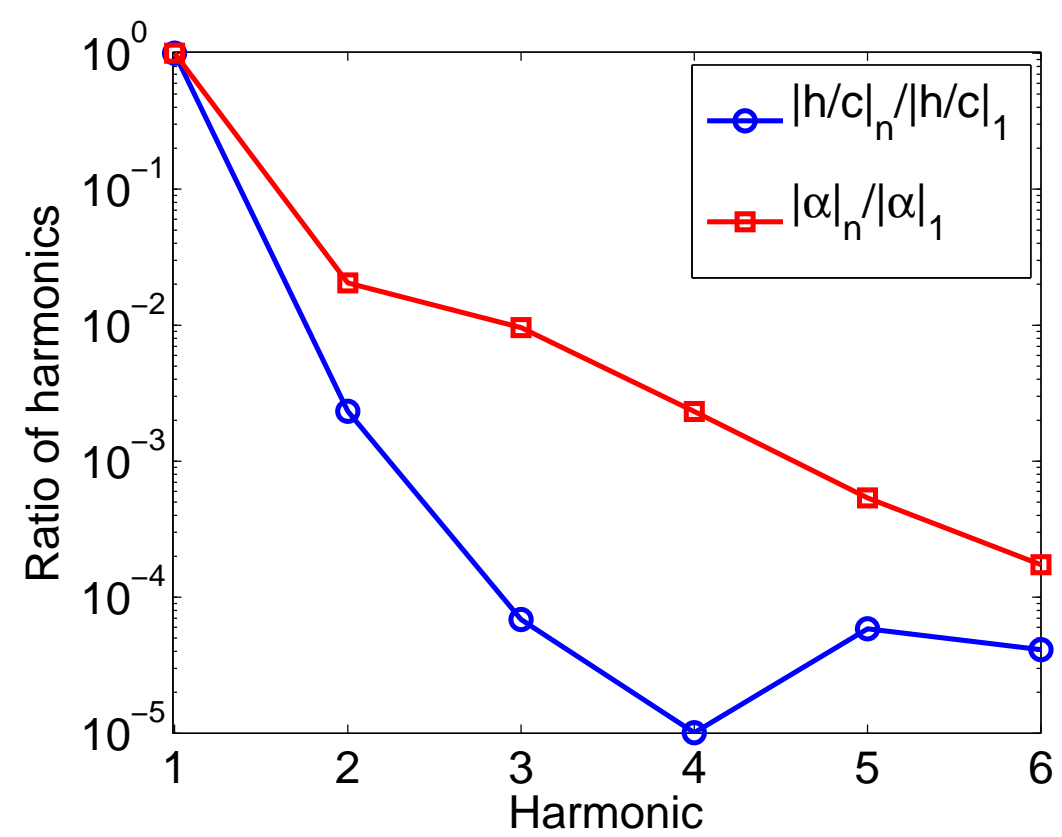

Figure 8: Ratio of higher harmonics to first harmonic of the LCO

first order harmonic, the LCO can be approximated using a single harmonic sinusoidal with reasonable accuracy. Further evidence that the first order harmonic is sufficient to model the LCOs considered in this work is given later on.

In order to analyse the development of an LCO it is helpful to determine the components of the power of the system. The individual contributions of the aerodynamic lift $\left(P_{l}\right)$, aerodynamic moment $\left(P_{m}\right)$ and structural damping $\left(P_{d}\right)$ to the total power $P_{\text {tot }}$ can be determined from:

$$
\begin{aligned}
P_{d} & =-D_{h} \dot{h}^{2}-D_{\alpha} \dot{\alpha}^{2}, \\
P_{l} & =-\dot{h} L, \\
P_{m} & =\dot{\alpha} M, \\
P_{\text {tot }} & =P_{d}+P_{l}+P_{m} .
\end{aligned}
$$

The aerodynamic power is the sum of the power of the lift and of the moment: $P_{\text {aero }}=P_{l}+P_{m}$. In case of an LCO the aerodynamic power $P_{\text {aero }}$ should compensate the structurally dissipated power $P_{d}$ on average. The power components have been obtained by multiplying the time signals of the aerodynamic forces and the motion vector $\vec{q}$. To obtain the mean power components, the instantaneous power is averaged over an oscillation period, in equation form:

$$
\bar{P}=\frac{1}{T} \int_{t}^{t+T} P d t,
$$

where $\bar{P}$ is the mean power and $T$ is the length of an oscillation period. When the instantaneous power signal is averaged a smooth curve of mean values of the power results. The oscillation amplitude at each time step has, in this case, been obtained by spline fitting through the maxima and minima of the pitch angle and the plunge displacement time signals.

Figure 9 shows the mean of the power components computed in this manner versus amplitude for simulation 1 (with an initial amplitude below the LCO amplitude) and simulation 2 (with an initial amplitude above the LCO amplitude). It is clearly seen that the total power becomes zero at an amplitude of $3.11^{\circ}$. This is therefore the LCO 


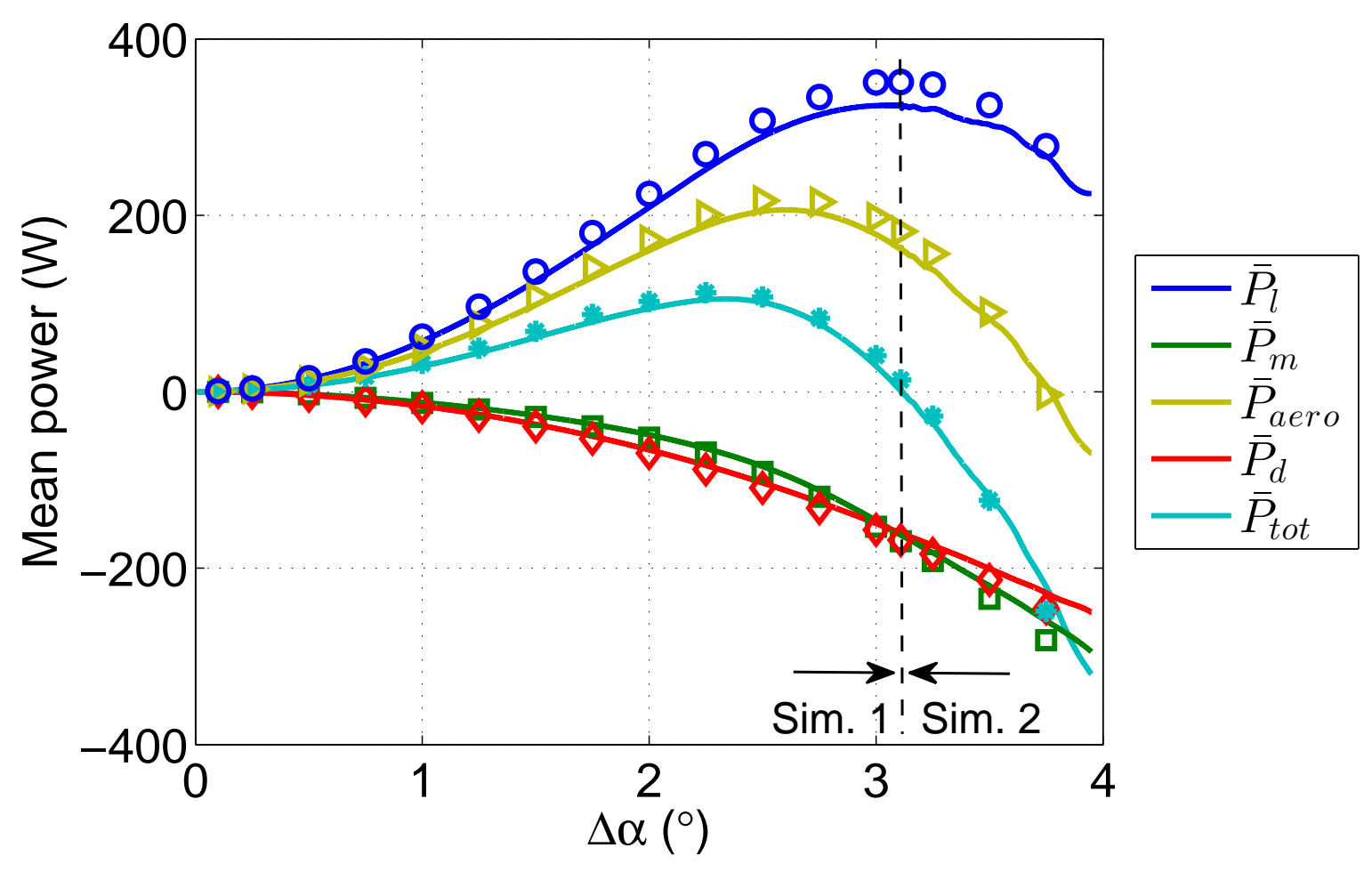

Figure 9: Mean power versus amplitude for LCO at $M=0.74, \bar{\alpha}=-0.8^{\circ}$ (FSI - lines and forced motion - symbols)

amplitude. The structural damping subtracts energy from the system. The same holds for the aerodynamic moment, whereas the lift adds energy to the system. The same behaviour was observed from the experimental results of Dietz et al. [4]. Note that they used different test cases and hence obtained different LCO amplitudes.

\subsection{Single harmonic forced motion oscillations simulations}

Figure 9 shows the power components versus the amplitude as obtained from the (reference) FSI simulation (lines) and from forced motion oscillations simulations with a complex-valued amplitude ratio (symbols). The complex amplitude ratio used for each LCO amplitude (i.e. the amplitude ratio and the phase difference) has been extracted from the FSI simulations. The phase difference between pitch and plunge changes slightly over time during the coupled simulation, the same holds for the amplitude ratio (see Figure 7). These changes have been taken into account in the forced motion simulations As the reduced frequency is constant during the LCO development, it has been fixed at 0.254 here. Table 2 shows the amplitudes at which forced motion oscillation simulations were performed together with the corresponding phase differences and amplitude ratios.

As can be seen from Figure 9 there is a good agreement between the power components obtained from the forced oscillations and those obtained from the FSI simulations. Hence, this suggests that taking into account the first order harmonic only is sufficient for modelling the LCO. The small deviations that appear in the power of the lift and in the aerodynamic power, are probably caused by the post-processing of the time signals of the FSI simulations, both in terms of the mode shape (see Figure 7 which shows a small band of solutions for both the amplitude ratio and the phase difference) and the power itself. A power analysis with low-pass filtered time signals, i.e. were only the first harmonics were retained, resulted in an only marginally smaller $\bar{P}_{l}$ and $\bar{P}_{\text {aero }}$. Hence, the higher harmonics in the coupled FSI simulations (which are not present in the forced motion simulations) are not the cause of the deviations between the mean power curves obtained from the forced oscillations and those obtained from FSI simulations. 


\begin{tabular}{c|c|c}
$\Delta \alpha\left(^{\circ}\right)$ & $\phi_{h \alpha}\left({ }^{\circ}\right)$ & $\left|\theta_{h \alpha}\right|$ \\
\hline \hline 0.0105 & 7.29 & 0.780 \\
0.1 & 7.32 & 0.780 \\
0.25 & 7.57 & 0.780 \\
0.5 & 8.06 & 0.779 \\
0.75 & 8.05 & 0.779 \\
1 & 8.50 & 0.778 \\
1.25 & 8.51 & 0.778 \\
1.5 & 8.41 & 0.779 \\
1.75 & 8.36 & 0.778 \\
2 & 8.30 & 0.779 \\
2.25 & 8.49 & 0.778 \\
2.5 & 8.66 & 0.779 \\
2.75 & 9.05 & 0.779 \\
3 & 9.46 & 0.779 \\
3.11 & 9.57 & 0.779 \\
3.25 & 9.73 & 0.779 \\
3.5 & 9.85 & 0.779 \\
3.75 & 9.90 & 0.779
\end{tabular}

Table 2: Phase difference and amplitude ratio used for forced motion oscillation simulations at $M=0.74, \bar{\alpha}=-0.8^{\circ}$

It should be noted here that variations of the phase difference $\phi_{h \alpha}$, which are possible when using forced motion oscillation simulations, have an enormous impact on the aerodynamic power and therefore on the LCO amplitude. Corresponding forced motion oscillation simulations, with a phase difference of zero, would lead to an incorrectly predicted LCO amplitude. Figure 10 demonstrates this by comparing the total power as obtained from forced motion oscillation simulations in case of a zero phase difference and in case of a non-zero phase difference. The symbols have been connected by interpolated splines. When the phase difference is zero an LCO with an amplitude of about $1.85^{\circ}$ would be obtained. This LCO amplitude is much smaller than the LCO amplitude actually obtained $\left(3.11^{\circ}\right)$. Hence, the phase difference, although small, can definitely not be ignored. This is in contrast to the observations of Dietz et al. [4] and Bendiksen [12], who suggested that the LCOs they observed during their experiments and computations, respectively, can be seen as a single-DOF motion. Note, however, that both studies conducted their investigations at different conditions and that Dietz et al. [4] observed significantly different LCO amplitudes.

\subsection{Non-linear aerodynamic power defect}

If the aerodynamic forces were proportional to the displacement, the power delivered by the lift would vary quadratically with amplitude. However, with increasing amplitude a non-linearity limits the (quadratic) increase of the power delivered by the lift, i.e. there exist a defect in the power of the lift (see Figure 9). The power of the moment is also a non-linear function of the amplitude, since it is coupled to the lift. Although, in comparison, the defect in the power of the moment is not as strong as for the power of the lift. These two effects enable the power of the structural damping to balance the aerodynamic power. However, the question is why do we have such a large defect in the power of the lift, i.e. what feature is responsible for this defect? And why is the defect so much smaller for the power of the moment?

To find the cause of this defect, one needs to look at the computation of the mean power of the lift, moment and damping. Assuming a harmonic input and a harmonic response, the mean of the power of the lift, moment and damping can be computed using: 


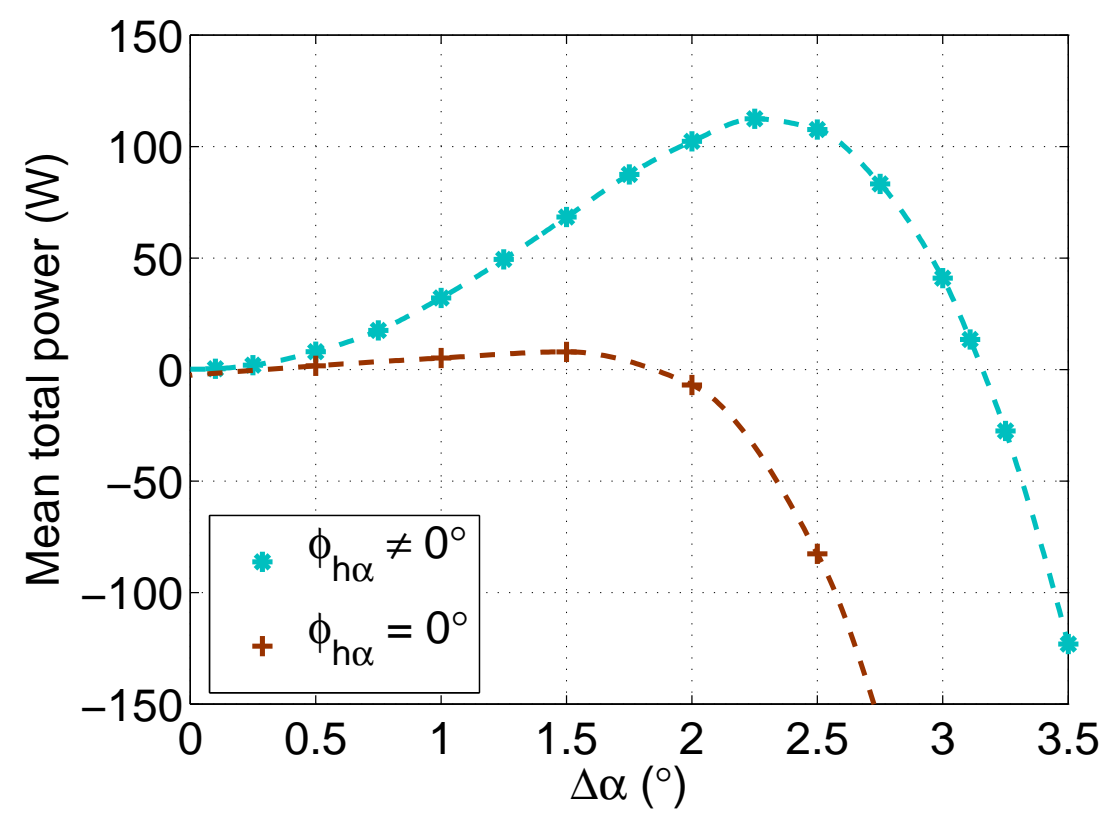

Figure 10: Mean total power versus amplitude for forced motion oscillations with zero and non-zero phase difference $\phi_{h \alpha}$ at $M=0.74, \bar{\alpha}=-0.8^{\circ}$

$$
\begin{aligned}
\bar{P}_{l} & =-0.5 \Delta h|L| \omega \sin \left(\phi_{L \alpha}-\phi_{h \alpha}\right)=-0.5 \Delta h|L| \omega \sin \phi_{L h}, \\
\bar{P}_{m} & =0.5 \Delta \alpha|M| \omega \sin \phi_{M \alpha}, \\
\bar{P}_{d} & =-0.5 \omega^{2}\left(D_{h}(\Delta h)^{2}+D_{\alpha}(\Delta \alpha)^{2}\right),
\end{aligned}
$$

where $|L|$ is the magnitude of the lift, $|M|$ is the magnitude of the moment, $\omega$ is the angular frequency, $\phi_{L \alpha}$ is the phase of the lift with respect to the pitching motion and $\phi_{M \alpha}$ is the phase of the moment with respect to the pitching motion. Subtracting $\phi_{h \alpha}$ from $\phi_{L \alpha}$ gives the phase of the lift with respect to the plunging motion $\phi_{L h}$.

Equations (12) and (13) are used to compute the equivalent linearised power components. The equivalent linearised power is the power that would have been obtained when the small amplitude mode shape and aerodynamic forces had been retained at large amplitudes, i.e. in the case of linear flutter. The equivalent linearised complex-valued aerodynamic lift and moment needed to compute these power components, are computed by scaling up the lift and moment from the small-amplitude values of FSI simulation 1. The phase of both lift and moment is just constant in the linearised case. Figure 11 shows the mean of the equivalent linearised power components versus oscillation amplitude (dashed lines). The power signals from the FSI simulations have been included in Figure 11 as well (solid lines). The equivalent linearised power of the lift $\tilde{P}_{l}$ increases with amplitude and until an amplitude of about $1.85^{\circ}$ it is approximately equal to $\bar{P}_{l}$. For larger amplitudes, $\bar{P}_{l}$ bursts and obtains a maximum. Similar behaviour is observed for the aerodynamic power and the total power. The equivalent linearised power of the moment $\tilde{P}_{m}$ does not decrease as fast as the non-linear power of the moment $\bar{P}_{m}$ with increasing amplitude. The power of the damping is the same in both cases, since it is computed from the structural properties of the system. Hence, Figure 11 clearly displays the non-linear aerodynamic power defect.

Now, why is there a defect in the power of lift and why is the defect in the power of the moment so much smaller? In order to answer these questions, one needs to look at the complex-valued ratios of the first harmonic components of the aerodynamic lift and moment during the time evolution of the LCO, i.e. $\theta_{L h}$ and $\theta_{M \alpha}$. The magnitude and phase angles of these complex-valued ratios of the lift and moment are shown in Figure 12. For comparison, the equivalent linearised magnitude and phase angle of the lift and moment have been included as well. Figure 12 shows that the 


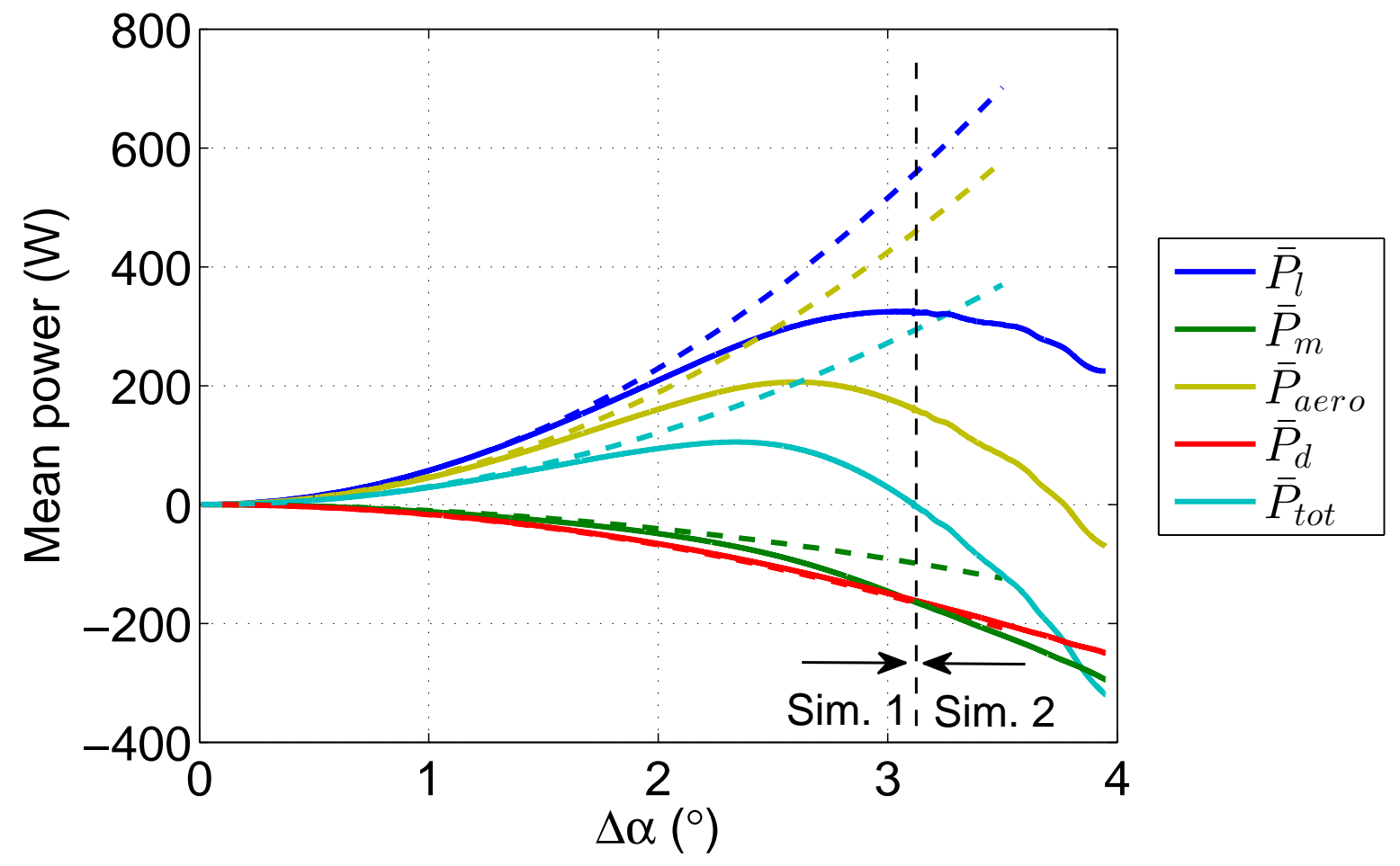

Figure 11: Mean power versus amplitude for LCO at $M=0.74, \bar{\alpha}=-0.8^{\circ}$ (FSI - full lines and equivalent linearised - dashed lines) 


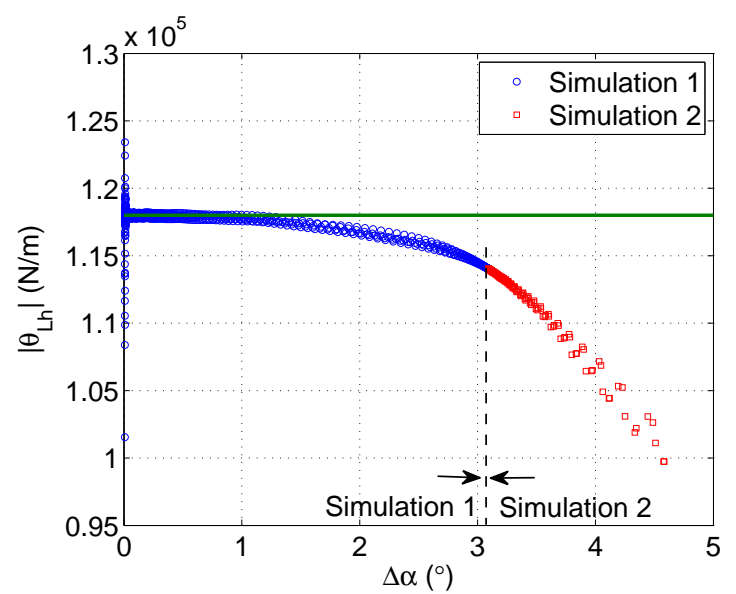

(a) $\left|\theta_{L h}\right|$ vs. $\Delta \alpha$

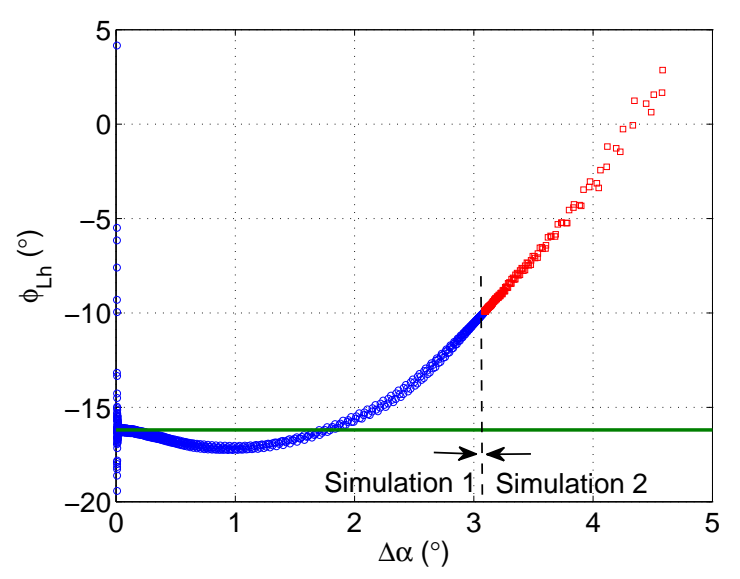

(c) $\phi_{L h}$ vs. $\Delta \alpha$

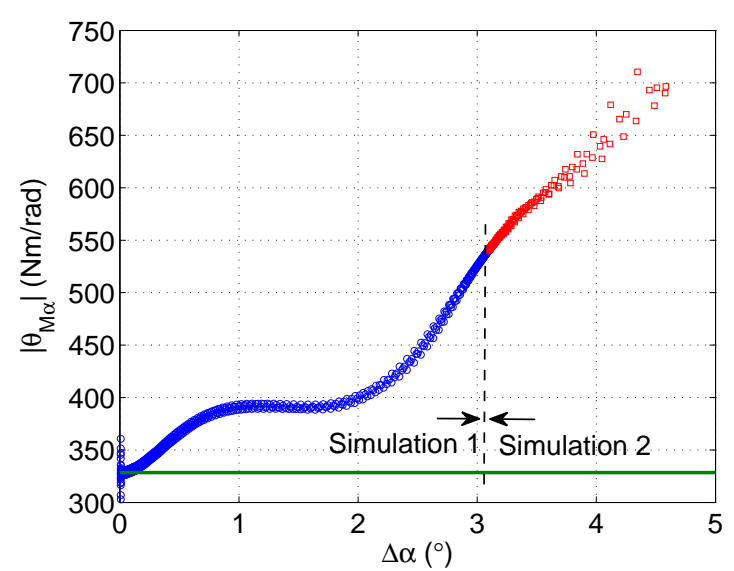

(b) $\left|\theta_{M \alpha}\right|$ vs. $\Delta \alpha$

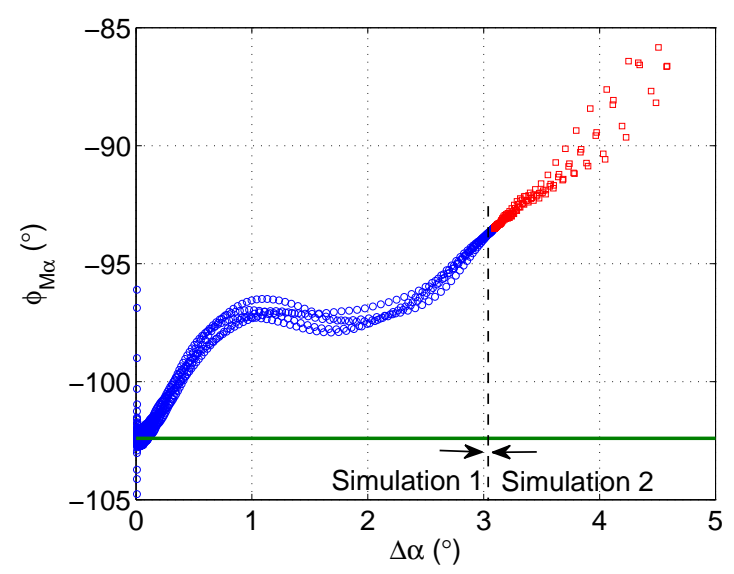

(d) $\phi_{M \alpha}$ vs. $\Delta \alpha$

Figure 12: Magnitude and phase angle of lift and moment versus amplitude for LCO at $M=0.74, \bar{\alpha}=-0.8^{\circ}$

magnitude of the lift during the LCO evolution $\left|\theta_{L h}\right|$ is almost the same in the equivalent linearised case. For the LCO amplitude a deviation of only $3 \%$ occurs. However, the phase of the lift $\phi_{L h}$ changes significantly. Furthermore, it is close to zero, hence the impact on the power of the lift will be enormous, since the sensitivity of the sine is the largest close to zero (see equation (12)). The magnitude of the moment $\left|\theta_{M \alpha}\right|$ deviates significantly from its equivalent linearised value at larger amplitudes. The phase of the moment $\phi_{M \alpha}$ is more negative in the equivalent linearised case than in the non-linear case. Deviations of about $5-10^{\circ}$ are present. However, since the phase of the moment is about $-90^{\circ}$, the impact on the power of the moment will not be as large as for power of the lift, since the slope of the sine is almost zero near $-90^{\circ}$ (see equation (13)).

To explain the non-linear defect of the power of the lift at higher amplitudes, equation (12) and Figures 12a and $12 \mathrm{c}$ are addressed. The power of the lift is a function of the plunge amplitude, this amplitude changes in the same way in both the equivalent linearised and the non-linear case. The frequency is also the same in both cases. Furthermore, the magnitude of the lift $\left|\theta_{L h}\right|$ was seen to be almost the same in both cases (see Figure 12a). That leaves the phase of the lift with respect to plunge $\phi_{L h}$ as a possible source of the defect. As observed before, the sine has a large sensitivity since $\phi_{L h}$ is close to zero. From Figure 12c it is seen that the difference between the phase of the lift in the linearised case and $\phi_{L h}$ starts to increase rapidly at an amplitude of approximately $1.85^{\circ}$, i.e. at the same amplitude as the power 
of the lift starts to deviate. Hence, the power of the lift shows a non-linear defect due to the change in the phase of the lift with increasing oscillation amplitude. This means that for an LCO to occur the phase of the lift has to change only slightly with oscillation amplitude, provided that the value of $\phi_{L h}$ is close to zero.

The defect in the power of the moment is explained in the same manner, only in this case a combination of the changes in $\left|\theta_{M \alpha}\right|$ and $\phi_{M \alpha}$ is responsible for this defect, since the phase of the moment is close to $-90^{\circ}$. Therefore, the change in magnitude and the change in phase of the moment with increasing amplitude are responsible for the power defect of the moment. These two defects, result in a defect in the aerodynamic power, which leads to the amplitude limitation of the oscillations.

To investigate what local features are responsible for the change in the complex-valued ratios of the first harmonic components of the aerodynamic lift an moment, the unsteady local force- and moment distributions at the LCO amplitude are compared to those obtained at $\Delta \alpha=0.01048^{\circ}$ (equivalent linearised condition). Figure 13a shows the steady surface pressure coefficient distribution at $M=0.74, \alpha=-0.8^{\circ}$. Figure $13 \mathrm{~b}$ depicts the imaginary part of the unsteady local lift distribution with respect to the plunging motion, i.e. the imaginary part of the complexvalued ratio of the first harmonic component of the local lift force $\mathfrak{J}\left(\theta_{L h}^{\text {local }}\right)$. The imaginary part of the unsteady local moment distribution with respect to the pitching motion (i.e. the imaginary part of the complex-valued ratio of the first harmonic component of the local moment $\mathfrak{J}\left(\theta_{M \alpha}^{\text {local }}\right)$ ) is shown in Figure 13c. The solid lines represent the LCO condition and the dashed lines the equivalent linearised condition. The upper surface is shown in blue and the lower surface in green. Note that Figure $13 \mathrm{~b}$ depicts the lift due to both pitch and plunge, it is merely referred to the plunging motion. The same holds for the local moment which is merely referred to the pitching motion in Figure 13c. The surface pressure coefficient distribution shows a strong pressure increase wave near the leading edge on the upper surface. However, this re-compression is not strong enough to re-compress the air such that the velocity downstream of it becomes subsonic. Instead the re-compression on the upper surface takes place without a shock near the midchord. On the lower surface a small pressure increase is also present near the leading edge. Downstream of it the flow expands and near $x / c=0.45$ a strong shock is present.

When looking at the imaginary part of the unsteady local lift $\mathfrak{J}\left(\theta_{L h}^{\text {local }}\right)$ it is observed that in the linearised case, several sharp peaks appear. All these peaks are related to unsteady shock motions since they are located at those locations where the steady surface pressure coefficient distribution exhibits a re-compression, re-compression shock or a small expansion. At the LCO condition these sharp peaks have disappeared and the local lift force is just smoothed out over the surface, i.e. on the lower surface the shock moves over a larger distance of the chord. On the upper surface the amplitude is large enough to establish a shock over part of the oscillation cycle, hence the wide shock peak at about mid-chord at the LCO condition. This peak and the difference in the imaginary part of the local lift force downstream of this peak cause the decrease in imaginary part of the lift from the linear condition to the LCO condition (see Figure $12 \mathrm{a}$ and $12 \mathrm{c})$.

The dynamics and the formation of shock waves also change the power of the moment significantly. This can be explained by looking at the imaginary part of the local moment distribution with respect to the pitch amplitude, $\mathfrak{J}\left(\theta_{M \alpha}^{\text {local }}\right)$, see Figure 13c. This distribution is similar to that of the local lift, it is just weighted because of the moment arm. At $x / c=0.25$ the local moment is zero, since this is the elastic axis location. From about mid-chord to the trailing edge the absolute value of the local moment on the upper surface is much larger in the LCO case than in the equivalent linearised case. Near the trailing edge this also holds for the lower surface. This increases the area underneath the curves such that $\mathfrak{J}\left(\theta_{M \alpha}^{\text {local }}\right)$ is much larger in the non-linear case than in the linearised case. This corresponds to the observations from Figures $12 \mathrm{~b}$ and $12 \mathrm{~d}$.

From the instantaneous surface pressure coefficient distributions at several timesteps it is observed that the shocks that exist on the upper surface and lower surface at the LCO condition are of Tijdeman [19] type B, since all shocks disappear during part of the oscillation cycle. Hence, from the linearised to LCO condition the type of shock motion changes from continuous (type A) to intermittent (type B). This coincides with the findings of Bendiksen [6, 7], who found that amplitude non-linearities in the aerodynamic forces are the strongest when the type of shock motion changes and these non-linearities will inevitably lead to limit-cycle oscillations. Intermittent boundary layer separation is also present on both airfoil surfaces at the LCO condition. In contrast, in the linearised case the flow remains attached. Hence, it is likely that the flow separation affects the shock wave motions in the non-linear case. However, it is not clear how large this effect is. An LCO would probably have occurred in the absence of the boundary layer as well, solely due to the amplitude non-linearities of the aerodynamic forces. Therefore it is thought that the unsteady 


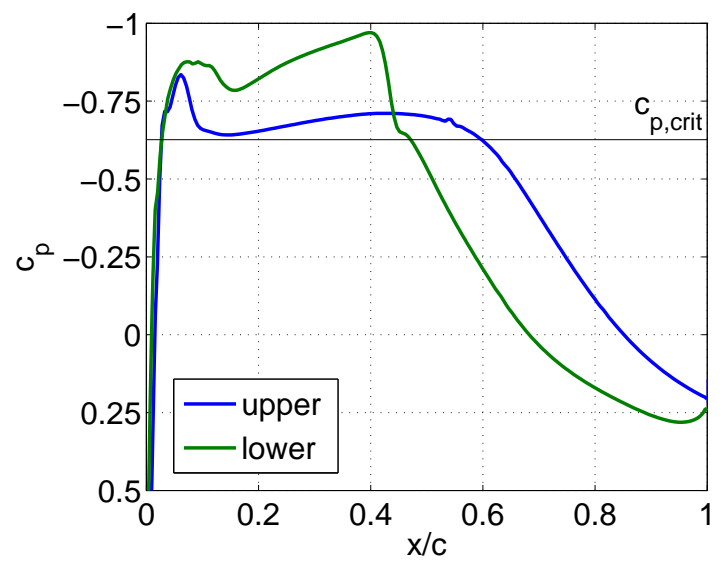

(a) Steady $c_{p}$ vs. $x / c$

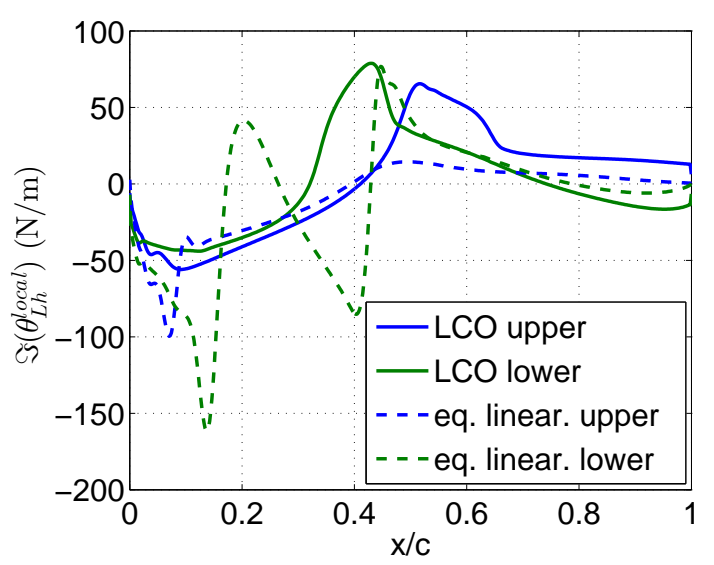

(b) $\mathfrak{J}\left(\theta_{L h}^{\text {local }}\right)$ vs. $x / c$

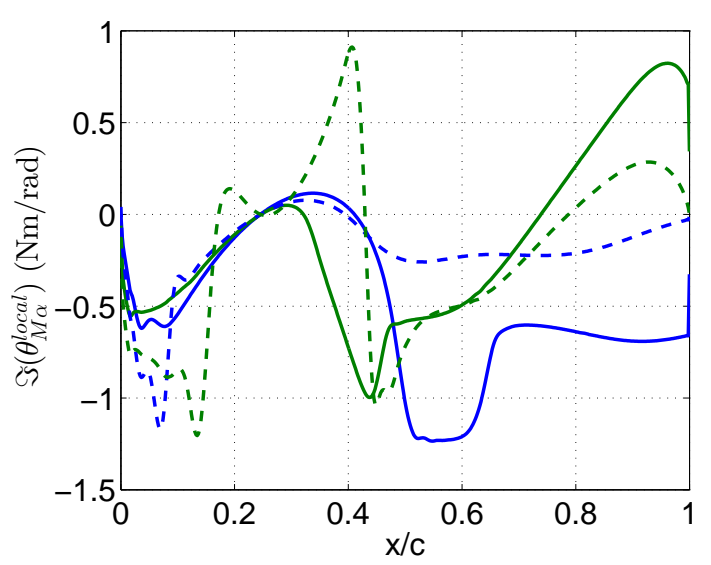

(c) $\mathfrak{J}\left(\theta_{M \alpha}^{\text {local }}\right)$ vs. $x / c$

Figure 13: Local force- and moment distribution for LCO and equivalent linear conditions at $M=0.74, \bar{\alpha}=-0.8^{\circ}$ 
shock wave motions are responsible for the variations in the magnitude and phase angle of the lift and the moment and hence for the non-linear defect in the aerodynamic power. However, the local trigger responsible for the amplitude limitation of the LCO studied in this work could not be identified due to the large amplitude range covered and hence the corresponding large shock movement.

\section{Conclusions}

Aerodynamic non-linearities that lead to the development of limit-cycle oscillations are a topic of current research. In this work the development and behaviour of LCOs were analysed using fluid-structure interaction simulations and harmonic forced motion oscillation simulations. These forced motion oscillations involved a sinusoidal oscillation at the fundamental frequency. From comparison with the FSI simulations it was observed that apparently the contribution of the higher harmonics is not significant for the LCOs considered in this work.

A power analysis of the coupled simulations showed that the slightly non-linear behaviour of the aerodynamic power enabled the occurrence of a limit-cycle oscillation. The power components in case of a limit-cycle oscillation were compared to the equivalent linearised power components that would exist in case of flutter. The defect of the power of the lift in the non-linear case was found to be caused by the changes in the phase angle of the lift with respect to plunge with oscillation amplitude. This phase angle was found to be close to zero and therefore very sensitive to small changes. The defect in the power of the moment was found to be much smaller than in case of the lift. In case of the moment, variations in both magnitude and phase were found to be responsible for the defect in the power. However, since the phase of the moment is close to $-90^{\circ}$, small variations of this phase, caused by oscillation amplitude variations, do not have a large influence on the power of the moment. Hence, for the LCOs studied in this work, the amplitude limitation is mainly caused by the change in the phase of the lift with oscillation amplitude. The changes in the magnitude and phase angle of the lift and moment with oscillation amplitude were found to be caused by unsteady shock wave motions.

\section{References}

[1] G. Schewe, H. Deyhle, Experiments on transonic flutter of a two-dimensional supercritical wing with emphasis on the non-linear effects, London, UK, 1996, proceedings RAeS Conference on Unsteady Aerodynamics.

[2] A. Knipfer, G. Schewe, Investigations of an oscillating supercritical 2d wing section in a transonic flow, no. A99-16518, Deutsches Zentrum für Luft- und Raumfahrt, Reno, NV, 1999, 37th AIAA Aerospace Sciences Meeting and Exhibit.

[3] G. Schewe, H. Mai, G. Dietz, Nonlinear effects in transonic flutter with emphasis on manifestations of limit cycle oscillations, Journal of Fluids and Structures 18 (2003) 3-22.

[4] G. Dietz, G. Schewe, H. Mai, Experiments on heave/pitch limit-cycle oscillations of a supercritical airfoil close to the transonic dip, Journal of Fluids and Structures 19 (2004) 1-16.

[5] G. Dietz, G. Schewe, H. Mai, Amplification and amplitude limitation of heave/pitch limit-cycle oscillations close to the transonic dip, Journal of Fluids and Structures 22 (2006) 505-527.

[6] O. Bendiksen, Transonic limit cycle flutter/lco, no. AIAA 2004-1694, Univerisity of California, Los Angeles, Palm Springs, California, 2004, 45th AIAA/ASME/ASCE/AHS/ASC Structures, Structural Dynamics, and Materials Conference.

[7] O. Bendiksen, Influence of shocks on transonic flutter of flexible wings, no. AIAA 2009-2313, University of California, Los Angeles, Palm Springs, California, 2009, 50th AIAA/ASME/ASCE/AHS/ASC Structures, Structural Dynamics, and Materials Conference.

[8] S. Weber, K. Jones, J. Ekaterinaris, M. Platzer, Transonic flutter computations for a 2d supercritical wing, no. A99-16647, Naval Postgraduate School, Monterey, CA, Reno, NV, 1999, 37th AIAA/ASME/ASCE/AHS/ASC Structures, Structural Dynamics, and Materials Conference and Exhibit.

[9] B. Castro, K. Jones, J. Ekaterinaris, M. Platzer, Analysis of the effect of porous wall interference on transonic airfoil flutter, Naval Postgraduate School, Monterey, CA, Anaheim, CA, 2001, 31st AIAA Fluid Dynamics Conference \& Exhibit.

[10] L. Tang, R. Bartels, P. Chen, D. Liu, Numerical investigation of transonic limit cycle oscillations of a two-dimensional supercritical wing, Journal of Fluids and Structures 17 (2003) 29-41.

[11] B. Wang, G.-C. Zha, Numerical simulation of transonic limit cycle oscillations using high-order low-diffusion schemes, Journal of Fluids and Structures 26 (2010) 579-601.

[12] O. Bendiksen, Role of shock dynamics in transonic flutter, AIAA Paper 92-2121-CP (1992).

[13] B. Stickan, J. Dillinger, G. Schewe, Computational aeroelastic investigation of a transonic limit-cycle-oscillation experiment at a transport aircraft wing model, Journal of Fluids and Structures 49 (2014) 223-241.

[14] T. Gerhold, M. Galle, Calculation of complex three-dimensional configurations employing the DLR TAU-code., AIAA Paper 97-0167.

[15] A. Jameson, W. Schmidt, E. Turkel, Numerical solutions of the Euler equations by finite volume methods using Runge-Kutta time-stepping schemes, AIAA Paper 1981-1259, 1981.

[16] A. Jameson, Time dependent calculations using multigrid, with applications to unsteady flows past airfoils and wings, AIAA Paper 911596AIAA 10th Computational Fluid Dynamics Conference. 
[17] J. Zwaaneveld, Nlr 7301 airfoil, in: EXPERIMENTAL DATA BASE FOR COMPUTER PROGRAM ASSESSMENT - Report of the Fluid Dynamics Panel Working Group 04, no. AGARD-AR-138, 1979, pp. A4-1-A4-22.

[18] H. Hassig, An approximate true damping solution of the flutter equation by determinant iteration, Journal of Aircraft 8 (11).

[19] H. Tijdeman, Investigations of the transonic flow around oscillation airfoils, Ph.D. thesis, Technische Hogeschool Delft (1977). 